\title{
FREQUENCY RESPONSE BASED CURVE FITTING APPROXIMATION OF FRACTIONAL-ORDER PID CONTROLLERS
}

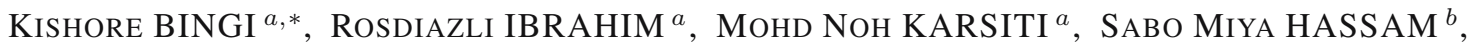 \\ VIVEKANANDA RAJAH HARINDRAN ${ }^{c}$
}

\begin{abstract}
${ }^{a}$ Department of Electrical and Electronics Engineering
PETRONAS University of Technology, Seri Iskandar, 32610 Perak, Malaysia e-mail: \{bingi.sai_g03426, rosdiazli, nohka\}@utp.edu.my
\end{abstract}

\author{
${ }^{b}$ Department of Electrical and Electronics Engineering \\ Abubakar Tafawa Balewa University, PMB 0248 Bauchi, Nigeria \\ e-mail: smhassan@atbu.edu.ng \\ ${ }^{c}$ Instrumentation and Control
}

PETRONAS Group Technical Solutions, Jalan Sultan Hishamuddin, 50050 Kuala Lumpur, Malaysia

e-mail: V_rharindran@petronas.com

\begin{abstract}
Fractional-order PID (FOPID) controllers have been used extensively in many control applications to achieve robust control performance. To implement these controllers, curve fitting approximation techniques are widely employed to obtain integer-order approximation of FOPID. The most popular and widely used approximation techniques include the Oustaloup, Matsuda and Cheraff approaches. However, these methods are unable to achieve the best approximation due to the limitation in the desired frequency range. Thus, this paper proposes a simple curve fitting based integer-order approximation method for a fractional-order integrator/differentiator using frequency response. The advantage of this technique is that it is simple and can fit the entire desired frequency range. Simulation results in the frequency domain show that the proposed approach produces better parameter approximation for the desired frequency range compared with the Oustaloup, refined Oustaloup and Matsuda techniques. Furthermore, time domain and stability analyses also validate the frequency domain results.
\end{abstract}

Keywords: curve fitting, fractional-order PID controller, frequency response, integer-order approximation, Oustaloup approximation, Matsuda approximation.

\section{Introduction}

In industrial process control applications, PID controllers are most widely employed for low-level control (Bingi et al., 2018a). This is because they are simple to design and easy to tune, and their implementation is seamless (Pachauri et al., 2018; Bingi et al., 2018b; Shah and Agashe, 2016). The fractional-order PID (FOPID or $\mathrm{PI}^{\lambda} \mathrm{D}^{\mu}$ ) controller is a variant of PID realized through fractional-ordering of the integral and derivative actions (Monje et al., 2010; Xue et al., 2007; Shah and Agashe, 2016; Xue, 2017). A key feature of $\mathrm{PI}^{\lambda} \mathrm{D}^{\mu}$ is that it is robust to system parameter variations and provides

${ }^{*}$ Corresponding author stable performance, especially for higher-order systems. Moreover, the controller can easily attain the iso-damping property (Monje et al., 2010; Shah and Agashe, 2016). It should be noted that seven different configurations can be achieved with the $\mathrm{PI}^{\lambda} \mathrm{D}^{\mu}$ controller (i.e., P, PI, $\mathrm{PI}^{\lambda}, \mathrm{PD}, \mathrm{PD}^{\mu}$, PID and $\mathrm{PI}^{\lambda} \mathrm{D}^{\mu}$ ) (Xue et al., 2007; Xue, 2017; Monje et al., 2010; Kishore et al., 2018; Shah and Agashe, 2016). However, a key issue with the practical realization or equivalent circuit implementation of such controllers in a finite-dimensional integer-order system is the approximation of the fractional-order parameters. This has generated a lot of interest among researchers recently.

For effective approximation of a fractional-order integrator and differentiator in the $\mathrm{PI}^{\lambda} \mathrm{D}^{\mu}$ controller, 
researchers have proposed several frequency domain approximation techniques (Krishna, 2011; Vinagre et al., 2000; Li et al., 2017). Despite these proposals, it is very difficult to determine the best method. This is because, while considering certain conditions such as the order of approximation or the accuracy of frequency and time responses, some of these methods can be more advantageous over others (de Oliveira Valério, 2005; Djouambi et al., 2007; Deniz et al., 2016).

Among the frequency domain approximation techniques available in the literature, the Oustaloup approximation is most popular and widely used. It is based on a recursive distribution of poles and zeros in the desired frequency range using frequency response fitting (Merrikh-Bayat, 2012). In certain situations, the Oustaloup approximation allows fitting the entire frequency range of interest (Oustaloup et al., 2000; Monje et al., 2010). Thus, to overcome this drawback, a modified or refined version has been proposed (Merrikh-Bayat, 2012; Xue et al., 2006; Meng and Xue, 2012; Wei et al., 2014a; Krajewski and Viaro, 2014; Atherton et al., 2014). However, it produces a higher-order approximated model.

Subsequently, to reduce the order of approximation, Liang et al. (2014) proposed a fixed-pole approximation technique. Similarly, Carlson derived a different technique (Tepljakov et al., 2012) using the Newton iterative method for continued fractional expansion (CFE) of the fractional-order differentiator. However, this technique is limited to fewer values of the fractional-order parameter (Tepljakov et al., 2012; Sheng et al., 2011). Later, Matsuda proposed an approximation technique using the gain of the fractional-order transfer function (Valério et al., 2013). However, in this method, if the order of approximation is chosen as an odd number, the approximated transfer function will be improper; i.e., there will be one more zero than poles (Yüce et al., 2017; Vinagre et al., 2000; Sheng et al., 2011). Other researchers have also proposed various power series expansion (PSE) techniques based on Taylor series, Maclaurin series, etc. (Valério et al., 2013; Petráš, 2011a; 2011b; Caponetto, 2010). Nevertheless, it is proven that CFE methods are converging more rapidly than PSE ones (Vinagre et al., 2000).

In the related development, Charef proposed an approximation technique (Das, 2011) where the accuracy is determined by properly selecting the maximal permissible error. However, the order of approximation involves a significant amount of trials and errors (Mitkowski and Oprzedkiewicz, 2016; Oprzedkiewicz, 2014). Thus, an extension of this method was proposed by Meng and Xue (2012). This extended method is focused on improving the accuracy of the original proposal. Other approximation algorithms based on the stability boundary locus (Deniz et al., 2016), the vector fitting method (Du et al., 2017), the time moments approach (Khanra et al., 2013), the state space approach (Poinot and Trigeassou, 2003; Krajewski and Viaro, 2011) and the frequency distribution mode (Wei et al., 2014b) have been proposed, too. A key issue with these methods is that they are quite complex and hence difficult to implement.

Motivated by the discussion above, this paper proposes a simple curve fitting approximation approach using exact frequency response data of fractional-order operators (differentiator/integrator). The proposed approach is expected to achieve better approximation compared with the commonly used Oustaloup, refined Oustaloup and Matsuda techniques (Monje et al., 2010). To demonstrate the performance of the proposed approach, a simulation study will be conducted on a class of fractional-order based controllers and systems.

The remaining sections of the paper are organized as follows: the definitions of the fractional-order differintegral operator and the fractional-order PID controller as well as an overview of the Oustaloup, refined Oustaloup and Matsuda approximation algorithms are presented in Section 2. The proposed curve fitting approximation using the frequency response and the integer-order approximation table for a fractional-order differentiator are given in Section 3. A simulation study on fractional-order based controllers and systems to demonstrate the performance of the proposed approximation is given in Section 4 Finally, Section 5 concludes the paper.

\section{Preliminaries}

This section is divided into two parts. The first will briefly discuss fractional calculus while the second will give an overview of some standard approximations.

2.1. Fractional calculus. In this subsection, the definitions of the fractional-order differintegral operator and the fractional-order PID controller are presented.

2.1.1. Fractional-order differintegral. In fractional calculus, the fractional-order differintegral operator, a combined fractional-order differentiator and integrator, which generalizes the notation for the differentiator $(\operatorname{Re}(\gamma)>0)$ and the integrator $(\operatorname{Re}(\gamma)<0)$ for the function $x(t)$ (Kaczorek, 2018; Joice Nirmala and Balachandran, 2017), is defined as

$$
{ }_{a} \mathcal{D}_{T}^{\gamma} x(t)= \begin{cases}\int_{0}^{t} x(\tau) \mathrm{d} \tau^{\gamma}, & \operatorname{Re}(\gamma)<0, \\ x(t), & \operatorname{Re}(\gamma)=0, \\ \frac{\mathrm{d}^{\gamma} x(t)}{\mathrm{d} t^{\gamma}}, & \operatorname{Re}(\gamma)>0,\end{cases}
$$

where 
- $T$ and $a$ are the lower and upper bounds of the operator,

- $\gamma$ is the order of the operator, and

- $\operatorname{Re}(\gamma)$ is the real part of $\gamma$.

The Laplace transform of (11) at zero initial conditions is given as

$$
L\left\{{ }_{0} \mathcal{D}_{T}^{\gamma} x(t) ; s\right\}=s^{\gamma} X(s) .
$$

From (2), the approximation of the fractional-order differentiator $\left(s^{\gamma}\right)$ for $\gamma>0$ and the fractional-order integrator $\left(1 / s^{\gamma}\right)$ for $\gamma<0$ will be made using proposed curve fitting approach and other approximation techniques in Section 3 .

\subsubsection{Fractional-order PID controller (FOPID} or $\left.\mathbf{P I}^{\boldsymbol{\lambda}} D^{\mu}\right)$. The generalized transfer function of the $\mathrm{PI}^{\lambda} \mathrm{D}^{\mu}$ controller as reported by Shah and Agashe (2016) is

$$
C(s)=K_{p}+\frac{K_{i}}{s^{\lambda}}+K_{d} s^{\mu}, \quad 0<\lambda, \mu<2,
$$

where

- $K_{p}, K_{i}$ and $K_{d}$ are the proportional, integral and derivative constant gains,

- $\lambda$ is the order of integration, and

- $\mu$ is the order of differentiation.

From (3), the approximation of the fractional-order integrator $\left(1 / s^{\lambda}\right)$ and the fractional-order differentiator $\left(s^{\mu}\right)$ will be performed using the proposed curve fitting approach and other approximation techniques in Section 3 .

2.2. Overview of some standard approximation algorithms. In this subsection, three of the standard frequency domain approximation algorithms for the fractional-order differentiator $\left(s^{\gamma}\right)$ are presented. The approximation algorithms considered are the Oustaloup, refined Oustaloup and Matsuda ones. The performance of the proposed curve fitting technique will be compared these algorithms in Sections 3 and 4.

2.2.1. Oustaloup approximation. The Oustaloup approximation of the fractional-order differentiator $\left(s^{\gamma}\right)$ in the desired frequency range $\left(\omega_{l}, \omega_{h}\right)$ as defined by Oustaloup et al. (2000) is

$$
s^{\gamma} \approx \omega_{h}^{\gamma} \prod_{k=1}^{N} \frac{s+\omega_{k}^{\prime}}{s+\omega_{k}}, \quad 0<\gamma<1,
$$

with the zeros $\omega_{k}^{\prime}$ and poles $\omega_{k}$ of (4) computed as

$$
\begin{aligned}
& \omega_{k}^{\prime}=\omega_{l}\left(\frac{\omega_{h}}{\omega_{l}}\right)^{\frac{2 k-1-\gamma}{2 N}}, \\
& \omega_{k}=\omega_{l}\left(\frac{\omega_{h}}{\omega_{l}}\right)^{\frac{2 k-1+\gamma}{2 N}},
\end{aligned}
$$

where

- $\gamma$ is the order of the fractional-order derivative,

- $N$ is the order of approximation, and

- $\left(\omega_{l}, \omega_{h}\right)$ is the frequency range of interest.

The Oustaloup approximation is the most widely used technique for integer-order approximation of fractional-order operators. However, for practical applications, it is frequently found that it cannot fit the whole expected range of frequency (Monje et al., 2010; Xue et al., 2006; 2007).

2.2.2. Refined Oustaloup approximation. The modified or refined Oustaloup approximation of $s^{\gamma}$ in the desired frequency range $\left(\omega_{l}, \omega_{h}\right)$ is defined as

$$
\begin{aligned}
s^{\gamma} \approx & \left(\frac{d \omega_{h}}{b}\right)^{\gamma}\left(\frac{d s^{2}+b \omega_{h} s}{d(1-\gamma) s^{2}+b \omega_{h} s+d \gamma}\right) \\
& \times \prod_{k=-N}^{N} \frac{s+\omega_{k}^{\prime}}{s+\omega_{k}}, \quad 0<\gamma<1,
\end{aligned}
$$

with $\omega_{k}^{\prime}$ and $\omega_{k}$ being the respective zeros and poles computed as

$$
\begin{aligned}
& \omega_{k}^{\prime}=\omega_{l}\left(\frac{\omega_{h}}{\omega_{l}}\right)^{\frac{2 k-1-\gamma}{2 N}}, \\
& \omega_{k}=\omega_{l}\left(\frac{\omega_{h}}{\omega_{l}}\right)^{\frac{2 k-1+\gamma}{2 N}},
\end{aligned}
$$

where $b$ and $d$ are constants with values set as 10 and 9, respectively, to achieve good approximation (Xue et al., 2007). This modified approximation has very high accuracy in the entire frequency range (Xue et al., 2006). However, the method results in a very high integer-order transfer function.

2.2.3. Matsuda approximation. The Matsuda approximation of $s^{\gamma}$ will be performed in two steps. First, a rational model of $s^{\gamma}$ will be obtained using the continued fraction expansions (CFE) method. Then, the fitting of the original function at desired frequency points $\omega_{0}, \omega_{1}$, $\ldots, \omega_{n}$ is performed. Thereby, the approximated transfer function of $s^{\gamma}$ is

$$
s^{\gamma} \approx d_{0}\left(\omega_{0}\right)+\frac{s-\omega_{0}}{d_{1}\left(\omega_{1}\right)+\frac{s-\omega_{1}}{d_{2}\left(\omega_{2}\right)+\frac{s-\omega_{2}}{\cdots}}},
$$


where

$$
\begin{aligned}
d_{0}(\omega)= & \left|(j \omega)^{\gamma}\right|, \\
d_{k+1}(\omega)= & \frac{\omega-\omega_{k}}{d_{k}(\omega)-d_{k}\left(\omega_{k}\right)}, \\
& \quad k=0,1, \ldots, N .
\end{aligned}
$$

In this method, the sum of the total number of zeros and poles is known as the order of approximation $N$. Here, $N$ should be an even number; otherwise, the approximation technique results in an improper transfer function (Vinagre et al., 2000; Yüce et al., 2017; Deniz et al., 2016).

\section{Proposed curve fitting approximation}

The first stage of the proposed curve fitting approximation is obtaining the frequency response data ( $\mathrm{frd}$ ) of $s^{\gamma}$. This is accomplished by substituting $s=j \omega$ and then evaluating the function for different values of $\omega \in$ $\left(\omega_{l}, \omega_{h}\right)$ as follows:

$$
s^{\gamma}=\left.(j \omega)^{\gamma}\right|_{\omega=\left(\omega_{l}, \ldots, \omega_{h}\right)} .
$$

The next stage is to derive an integer-order transfer function model $G(s)$ from the data obtained in (13) using the Sanathanan-Koerner (SK) least-squares iterative method (Shi, 2016). Thus, the transfer function model of $G(s)$ is defined as follows:

$$
G(s)=\frac{P(s)}{Q(s)} \approx \frac{\sum_{n=0}^{N} p_{n} s^{n}}{1+\sum_{n=1}^{N} q_{n} s^{n}} \approx \frac{P \psi(s)}{1+Q \phi(s)},
$$

where the coefficients $P, Q$ and the monomial functions $\psi(s), \phi(s)$ are defined as follows:

$$
\begin{gathered}
P=\left[\begin{array}{c}
p_{0} \\
p_{1} \\
\vdots \\
p_{N}
\end{array}\right], \quad Q=\left[\begin{array}{c}
q_{1} \\
q_{2} \\
\vdots \\
q_{N}
\end{array}\right], \\
\psi(s)=\left[1, s, \ldots, s^{N}\right], \quad \phi(s)=\left[s, s^{2}, \ldots, s^{N}\right] .
\end{gathered}
$$

The objective is to identify $P$ and $Q$ in the integer-order transfer function $G(s)$ in the desired frequency range $\omega \in\left(\omega_{l}, \omega_{h}\right)$. This is achieved by minimizing the difference between data samples $(H(s))$ obtained from (13) and $G(s)$ using the following Levy linearized cost function with the SK least-squares iteration method:

$$
\arg \min _{P, Q} \sum_{k=l}^{h}\left|\frac{P\left(j \omega_{k}\right)}{Q^{\tau-1}\left(j \omega_{k}\right)}-\frac{Q^{\tau}\left(j \omega_{k}\right)}{Q^{\tau-1}\left(j \omega_{k}\right)} H\left(j \omega_{k}\right)\right|^{2},
$$

where $\tau=1, \ldots, \mathcal{T}$ is the iteration step. From (17), it should be noted that the unbiased curve fitting is achieved when $Q^{\tau-1}\left(j \omega_{k}\right)$ approaches $Q^{\tau}\left(j \omega_{k}\right)$. Furthermore, to obtain a minimal realization of $G(s)$, the matching poles and zeros will be cancelled.

The final stage is to convert the obtained state-space model given in (18) after pole-zero cancellation into a transfer function model of the following form:

$$
\begin{gathered}
s X(s)=A X(s)+B U(s), \\
Y(s)=C X(s)+D U(s), \\
G(s) \approx \frac{Y(s)}{U(s)}=C\left(s I_{N}-A\right)^{-1} B+D,
\end{gathered}
$$

where

- $X(s), Y(s)$ and $U(s)$ are the state, output and control vectors, respectively,

- $A, B, C$ and $D$ are the state, input, output, feedforward matrices, respectively.

Therefore, the above procedure for the proposed integer-order approximation of $s^{\gamma}$ based on the curve fitting of frequency response data with the use of MATLAB built-in commands will be implemented as follows:

1. Obtain the frequency response data for integer-order part of $s^{\gamma}$ within the desired frequency range $\omega \in\left(\omega_{l}\right.$, $\left.\omega_{h}\right)$ using the MATLAB inbuilt function $\mathrm{frd}($ ) .

2. Obtain the exact frequency response data of $s^{\gamma}$ by raising the data obtained in the previous step to a power of $\gamma$.

3. Choose the approximation order $N$ for the integer-order model.

4. Obtain the state space model of exact function response data based on SK's least-squares iteration method by using inbuilt MATLAB function fitfrd().

5. Convert the state space model to the transfer function using the inbuilt MATLAB command ss $2 \mathrm{tf}$ ( ) .

The MATLAB commands for implementing the proposed algorithm are provided in Appendix A To demonstrate the proposed approach, consider the fractional-order differentiator $s^{0.1}$. Here, the desired frequency range $\omega$ is chosen as $\left(10^{-2}, 10^{2}\right)$. Furthermore, to study the effect of variation in $N$, the order of approximation $N$ is chosen as 4,5 and 6 . The choice for the range of frequency and the order of approximation is based on works reported by Yüce et al. (2017), Meng and Xue (2012), Xue et al. (2007) and Deniz et al. 
(2016). Thus, the approximated transfer functions using the proposed approach for chosen orders are

$$
\begin{aligned}
& 1.714 s^{4}+75.22 s^{3}+248.1 s^{2}+83.38 s \\
& s^{0.1} \approx \frac{+1.935}{s^{4}+58.57 s^{3}+244.1 s^{2}+103.5 s} \\
& +3.237 \\
& 1.777 s^{5}+123.9 s^{4}+873.4 s^{3}+909.9 s^{2} \\
& s^{0.1} \approx \frac{+137.7 s+1.914}{s^{5}+90.81 s^{4}+785.4 s^{3}+985 s^{2}}, \\
& +182.9 s+3.335 \\
& 1.831 s^{6}+183.7 s^{5}+2279 s^{4}+5199 s^{3} \\
& s^{0.1} \approx \frac{+2427 s^{2}+203 s+1.9}{s^{6}+128.7 s^{5}+1919 s^{4}+5142 s^{3}} . \\
& +2818 s^{2}+283.7 s+3.424
\end{aligned}
$$

Consequently, the Bode plots of 20-22 are presented in Figs. 13 respectively. The plots are compared with the Oustaloup, refined Oustaloup and Matsuda approximations. From the figures, it can be observed that, for the orders of approximations 5 and 6 , the proposed approach is more accurate within the desired frequency range than the other methods.

To evaluate the effectiveness of the proposed approach, a time domain comparison was also performed. For this purpose, the exact step response of the fractional-order integrator $1 / s^{\gamma}$ is obtained from the inverse Laplace transform of the integer-order integrator $1 / s^{n}$ as

$$
L^{-1}\left[\frac{1}{s^{n}}\right]=\frac{t^{n-1}}{(n-1) !}, \quad n \in \mathbb{N} .
$$

As in the case of (23), the inverse Laplace transform of the fractional integrator $1 / s^{\gamma}$ is derived as

$$
L^{-1}\left[\frac{1}{s^{\gamma}}\right]=\frac{t^{\gamma-1}}{\Gamma(\gamma)}, \quad 0<\gamma<1,
$$

where $\Gamma(\gamma)=(\gamma-1)$ !. From (24), the step response is

$$
L^{-1}\left[\frac{1}{s^{\gamma+1}}\right]=\frac{t^{\gamma}}{\Gamma(\gamma+1)}=\frac{t^{\gamma}}{\gamma \Gamma(\gamma)} .
$$

This equation will be used to calculate the exact step response of the fractional order operator. Thus, the exact step response of $s^{0.1}$ is given by

$$
L^{-1}\left(s^{0.1}\right)=\frac{0.1 \times \Gamma(0.1)}{t^{0.1}} .
$$

The step responses of the proposed approach in comparison with the Oustaloup, refined Oustaloup and Matsuda approximations for the orders 4, 5 and 6, are
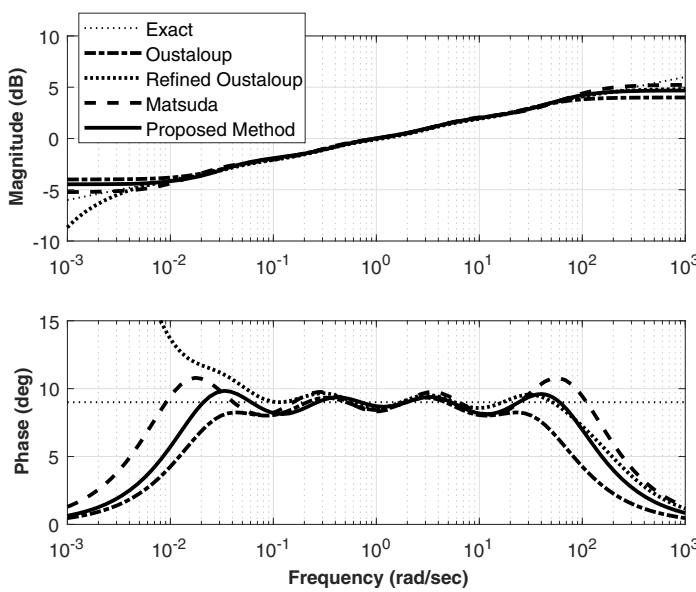

Fig. 1. Bode plots of the fractional-order differentiator $\left(s^{0.1}\right)$ for $N=4$ approximated using various methods.
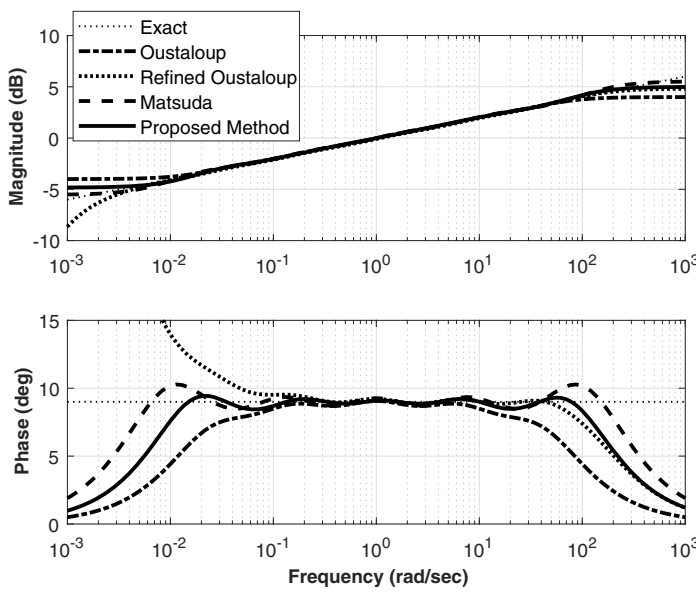

Fig. 2. Bode plots of the fractional-order differentiator $\left(s^{0.1}\right)$ for $N=5$ approximated using various methods.
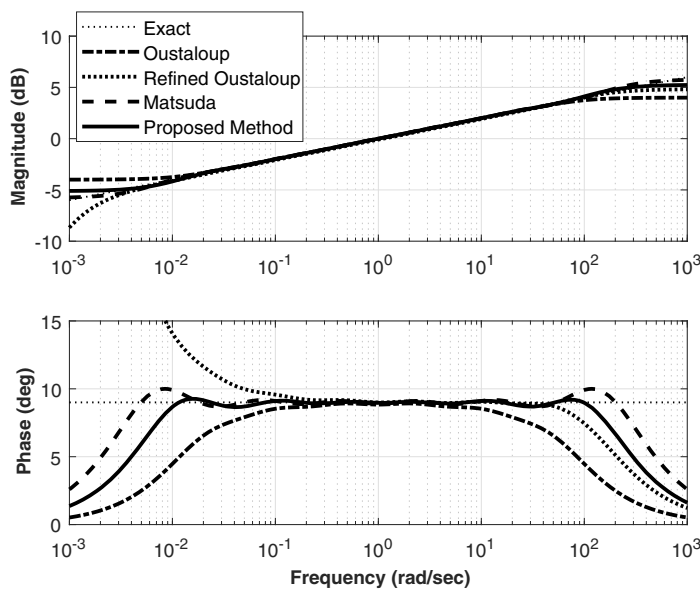

Fig. 3. Bode plots of the fractional-order differentiator $\left(s^{0.1}\right)$ for $N=6$ approximated using various methods. 
presented in Figs. 4, 5and 6 respectively. For numerical assessment of the results shown in the figures, the mean absolute error (MAE) is chosen

$$
\mathrm{MAE}=\frac{1}{n} \sum_{i=1}^{n}\left|y_{i}-x_{i}\right|,
$$

where

- $y_{i}$ is the approximated frequency response data,

- $x_{i}$ is the exact frequency response data, and

- $n$ is the total size of the data.

This type of error function is most suitable for non-time based problems (Deniz et al., 2016). Therefore, the numerical assessment of the responses is given in Table 1 . Observing the figures and the table, it can be seen that the proposed approach outperformed the compared techniques. The numerical analysis also confirmed that the proposed approach produced less error when compared with other approaches.

Furthermore, the stability analysis of the approximated transfer function $(G(s))$ will be made using $\mathrm{H}_{2}$ and $\mathrm{H}_{\infty}$-norms for the frequency band $\omega \in\left(\omega_{l}, \omega_{h}\right)$ as defined in (28) and (29), respectively. From the equations, it can be noted that, for a stable system, the $\mathrm{H}_{2}$-norm is the average system gain over all frequencies while the $\mathrm{H}_{\infty}$-norm is the peak gain of the frequency response. However, for an unstable system, these norms will be infinite:

$$
\begin{aligned}
\|G(s)\|_{2}= & \sqrt{\frac{1}{2 \pi} \int_{-\infty}^{\infty} \operatorname{trace}[G(j \omega) * G(j \omega)] \mathrm{d} \omega}, \\
& \|G(s)\|_{\infty}=\sup _{\omega \in\left(\omega_{l}, \omega_{h}\right)}|G(j \omega)| .
\end{aligned}
$$

Thus, the stability analysis of the proposed approach and other compared techniques from Table 1 shows that all the approximated transfer functions are stable with a finite value of the $\mathrm{H}_{\infty}$-norm.

The approximation table for the fractional-order differentiator $s^{\gamma}(\gamma=0.1,0.2, \ldots, 0.9)$ using the proposed approach is given in Table 2. The table can be used directly to obtain the approximated transfer function of fractional-order based systems and controllers. On the other hand, the numerical assessment of the approximation table for the step response of the proposed approach in comparison with the Oustaloup, refined Oustaloup and Matsuda techniques is given in Table 3 . From the table, it can be noted that for longer time periods, the proposed approach yields a better approximation than the other methods. Furthermore, observing the stability analysis in the table, it can also be noted that all the approximated transfer functions using various techniques are stable with a finite value of the $\mathrm{H}_{\infty}$-norm.

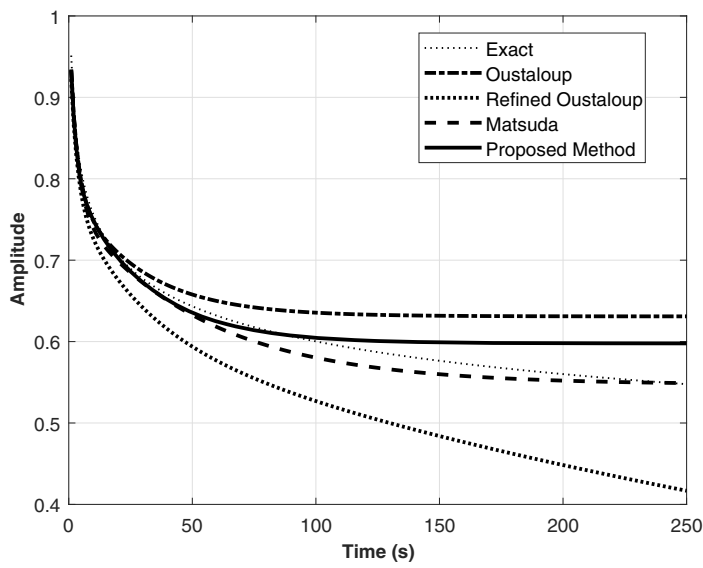

Fig. 4. Step responses of the fractional-order differentiator $\left(s^{0.1}\right)$ for $N=4$ approximated using various methods.

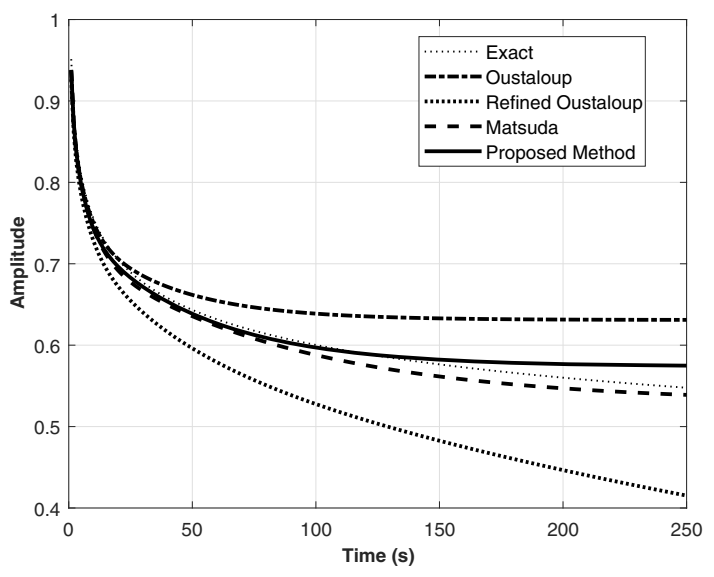

Fig. 5. Step responses of the fractional-order differentiator $\left(s^{0.1}\right)$ for $N=5$ approximated using various methods.

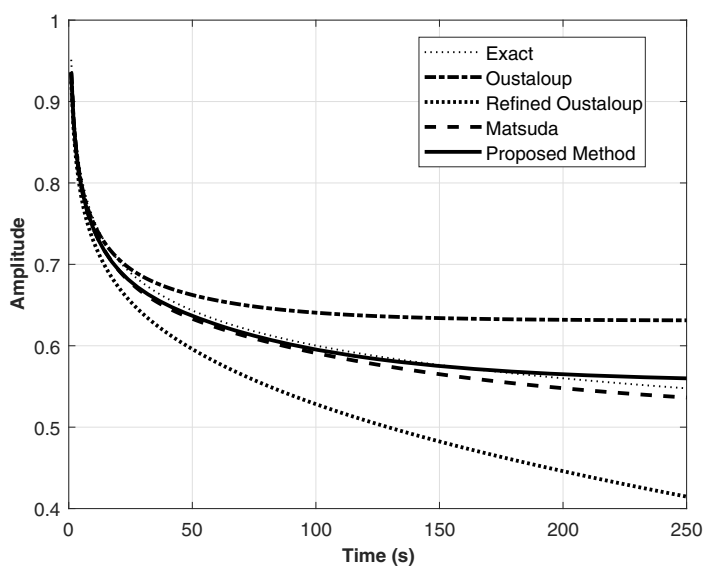

Fig. 6. Step responses of the fractional-order differentiator $\left(s^{0.1}\right)$ for $N=6$ approximated using various methods. 
Table 1. Numerical and stability analysis of the fractional-order differentiator $\left(s^{0.1}\right)$.

\begin{tabular}{|c|c|c|c|c|c|}
\hline Technique & Order & $t_{1} \in(0,125)$ & $t_{2} \in(0,250)$ & $\mathrm{H}_{\infty}$-norm & Stability \\
\hline \hline Oustaloup & & 0.0215 & 0.0441 & 1.5849 & stable \\
refined Oustaloup & \multirow{4}{*}{4} & 0.0547 & 0.0809 & 1.7425 & stable \\
Matsuda & & 0.0144 & 0.0122 & 1.8280 & stable \\
proposed & & 0.0061 & 0.0199 & 1.7145 & stable \\
Oustaloup & & 0.0237 & 0.0455 & 1.5849 & stable \\
refined Oustaloup & \multirow{2}{*}{5} & 0.0540 & 0.0813 & 1.7425 & stable \\
Matsuda & & 0.0110 & 0.0120 & 1.8905 & stable \\
proposed & & 0.0053 & 0.0097 & 1.7773 & stable \\
Oustaloup & & 0.0247 & 0.0463 & 1.5849 & stable \\
refined Oustaloup & \multirow{2}{*}{6} & 0.0538 & 0.0814 & 1.7425 & stable \\
Matsuda & & 0.0103 & 0.0110 & 1.9462 & stable \\
proposed & & 0.0071 & 0.0059 & 1.8315 & stable \\
\hline
\end{tabular}

Table 2. Approximations of fractional-order differentiators using the proposed curve fitting approach.

\begin{tabular}{|c|c|}
\hline$s^{\gamma}$ & Approximated transfer function \\
\hline \hline$s^{0.1}$ & $\frac{1.777 s^{5}+123.9 s^{4}+873.4 s^{3}+909.9 s^{2}+137.7 s+1.914}{s^{5}+90.81 s^{4}+785.4 s^{3}+985 s^{2}+182.9 s+3.335}$ \\
$s^{0.2}$ & $\frac{3.233 s^{5}+223 s^{4}+1624 s^{3}+1762 s^{2}+275.3 s+3.725}{s^{5}+116.8 s^{4}+1279 s^{3}+2011 s^{2}+473 s+11.14}$ \\
$s^{0.3}$ & $\frac{6.048 s^{5}+413.7 s^{4}+3111 s^{3}+3513 s^{2}+565.4 s+7.36}{s^{5}+151 s^{4}+2089 s^{3}+4115 s^{2}+1224 s+37.33}$ \\
$s^{0.4}$ & $\frac{11.7 s^{5}+794.7 s^{4}+6166 s^{3}+7236 s^{2}+1198 s+14.71}{s^{5}+197.2 s^{4}+3438 s^{3}+8478 s^{2}+3181 s+125.9}$ \\
$s^{0.5}$ & $\frac{23.59 s^{5}+1594 s^{4}+12740 s^{3}+15520 s^{2}+2632 s+29.63}{s^{5}+262.6 s^{4}+5753 s^{3}+17720 s^{2}+8368 s+429.6}$ \\
$s^{0.6}$ & $\frac{50.26 s^{5}+3381 s^{4}+27790 s^{3}+35050 s^{2}+6070 s+59.93}{s^{5}+361.4 s^{4}+9918 s^{3}+38090 s^{2}+22550 s+1498}$ \\
$s^{0.7}$ & $\frac{116 s^{5}+7769 s^{4}+65570 s^{3}+85390 s^{2}+15010 s+121.2}{s^{5}+526.7 s^{4}+18060 s^{3}+86240 s^{2}+63740 s+5453}$ \\
$s^{0.8}$ & $\frac{305.7 s^{5}+20410 s^{4}+176400 s^{3}+236400 s^{2}+41890 s+244.3}{s^{5}+857.9 s^{4}+36660 s^{3}+217000 s^{2}+199500 s+21830}$ \\
$s^{0.9}$ & $\frac{1092 s^{5}+72700 s^{4}+642000 s^{3}+881700 s^{2}+156000 s+489.3}{s^{5}+1852 s^{4}+98540 s^{3}+720500 s^{2}+819500 s+113800}$ \\
\hline
\end{tabular}

\section{Simulation study}

In this section, a simulation study will be conducted on five examples of fractional-order based controllers and systems. The selected systems are a differentiator, integrator, a PID controller, a low-pass filter and a higher-order transfer function. The obtained results from the proposed approach will be compared with those of the Oustaloup, refined Oustaloup and Matsuda approximations. Furthermore, the stability analysis of approximated transfer functions will be conducted using H-norms. In all the cases, the selected desired frequency range $\omega$ and the order of approximation $N$ are chosen as $\left(10^{-3}, 10^{3}\right)$ and 5 , respectively. As mentioned earlier, the choice for the range of frequency and order of approximation is based on the works reported by Yüce et al. (2017), Meng and Xue (2012), Xue et al. (2007) and Deniz et al. (2016). In addition, the order of $s^{\gamma}$ is limited to the $[-1,1]$ range for effective approximation (Valério et al., 2013). Thus, $s^{\gamma}$ is factorized as follows:

$$
s^{\gamma}=s^{\lfloor\gamma\rfloor} s^{\gamma-\lfloor\gamma\rfloor} \text {. }
$$

4.1. Fractional-order differentiator. This example demonstrates the approximation of a fractional-order differentiator

$$
G(s)=s^{0.26}
$$

using the proposed approach. A comparison with the exact solution and other approximation methods will also be given. Thus, the approximated transfer function using the proposed approach is

$$
\begin{aligned}
& 7.147 s^{5}+1836 s^{4}+2.493 \times 10^{4} s^{3} \\
& G(s) \approx+3.393 \times 10^{4} s^{2}+4811 s+29.74 \\
& s^{5}+597.2 s^{4}+1.608 \times 10^{4} s^{3} \\
&+3.85 \times 10^{4} s^{2}+1.022 \times 10^{4} s+163.4
\end{aligned}
$$

Similarly, the approximated transfer functions using other methods are given in Appendix B. 
Table 3. Numerical and stability analysis of approximation Table 2

\begin{tabular}{|c|c|c|c|c|c|}
\hline$s^{\gamma}$ & Technique & $t_{1} \in(0,125)$ & $t_{2} \in(0,250)$ & $\mathrm{H}_{\infty}$-norm & Stability \\
\hline \multirow{5}{*}{$s^{0.2}$} & Oustaloup & 0.0265 & 0.0510 & 2.5119 & stable \\
\hline & refined Oustaloup & 0.0832 & 0.1065 & 3.0744 & stable \\
\hline & Matsuda & 0.0286 & 0.0268 & 3.5911 & stable \\
\hline & proposed & 0.0208 & 0.0175 & 2.6816 & stable \\
\hline & Oustaloup & 0.0262 & 0.0450 & 3.9811 & stable \\
\hline \multirow{4}{*}{$s^{0.3}$} & refined Oustaloup & 0.0943 & 0.1045 & 5.5103 & stable \\
\hline & Matsuda & 0.0427 & 0.0363 & 6.8910 & stable \\
\hline & proposed & 0.0345 & 0.0233 & 4.4710 & stable \\
\hline & Oustaloup & 0.0272 & 0.0378 & 6.3096 & stable \\
\hline \multirow{4}{*}{$s^{0.4}$} & refined Oustaloup & 0.0942 & 0.0916 & 10.0820 & stable \\
\hline & Matsuda & 0.0511 & 0.0400 & 13.4464 & stable \\
\hline & proposed & 0.0433 & 0.0263 & 7.4974 & stable \\
\hline & Oustaloup & 0.0293 & 0.0319 & 10.0000 & stable \\
\hline \multirow{4}{*}{$s^{0.5}$} & refined Oustaloup & 0.0881 & 0.0761 & 18.9737 & stable \\
\hline & Matsuda & 0.0546 & 0.0398 & 26.9262 & stable \\
\hline & proposed & 0.0476 & 0.0270 & 12.7730 & stable \\
\hline & Oustaloup & 0.0314 & 0.0276 & 15.8489 & stable \\
\hline \multirow{4}{*}{$s^{0.6}$} & refined Oustaloup & 0.0794 & 0.0617 & 37.1951 & stable \\
\hline & Matsuda & 0.0548 & 0.0374 & 56.1234 & stable \\
\hline & proposed & 0.0488 & 0.0265 & 22.0811 & stable \\
\hline & Oustaloup & 0.0330 & 0.0256 & 25.1189 & stable \\
\hline \multirow{4}{*}{$s^{0.7}$} & refined Oustaloup & 0.0703 & 0.0496 & 77.7765 & stable \\
\hline & Matsuda & 0.0529 & 0.0340 & 124.8754 & stable \\
\hline & proposed & 0.0483 & 0.0245 & 38.8116 & stable \\
\hline & Oustaloup & 0.0338 & 0.0251 & 39.8107 & stable \\
\hline \multirow{4}{*}{$s^{0.8}$} & refined Oustaloup & 0.0618 & 0.0402 & 182.9633 & stable \\
\hline & Matsuda & 0.0499 & 0.0305 & 313.3221 & stable \\
\hline & proposed & 0.0468 & 0.0222 & 74.6020 & stable \\
\hline & Oustaloup & 0.0340 & 0.0205 & 63.0957 & stable \\
\hline \multirow{3}{*}{$s^{0.9}$} & refined Oustaloup & 0.0543 & 0.0330 & 573.8763 & stable \\
\hline & Matsuda & 0.0466 & 0.0273 & 1052.5 & stable \\
\hline & proposed & 0.0451 & 0.0249 & 150.9561 & stable \\
\hline
\end{tabular}

The Bode plots of the proposed approach in comparison with the Oustaloup, refined Oustaloup and Matsuda techniques are presented in Fig. 7 From the figure, it can be observed that the proposed approach is more accurate within the desired frequency range than for the other methods.

To evaluate the performance of the proposed approach, the step responses of all the compared techniques is shown in Fig. 8, while the numerical assessment of the figure is given in Table 4 In the figure, the exact time response of the system $G(s)$ obtained using (25) is

$$
g(t)=\frac{0.26 \times \Gamma(0.26)}{t^{0.26}}
$$

From both the figure and the table, it can be observed that, for the time period between 0 and 125 seconds, the Oustaloup technique has the least error of 0.0315 while, for the time period from 0 to 250 seconds, the
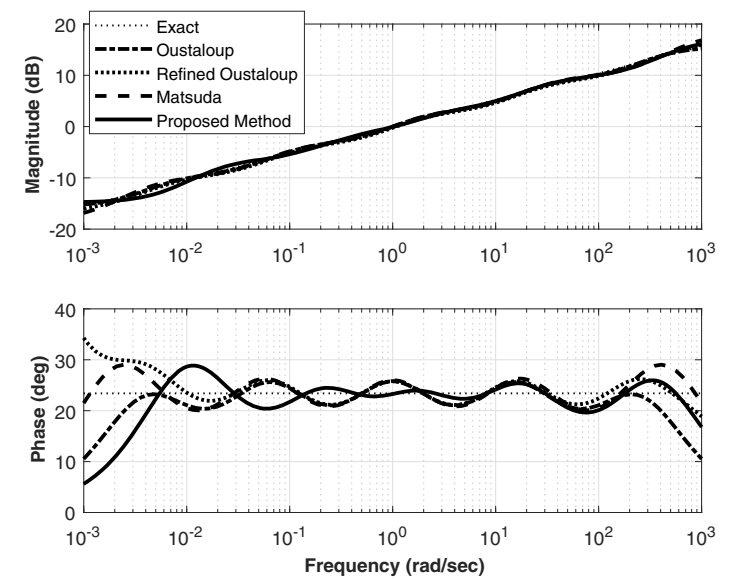

Fig. 7. Bode plots of the fractional-order differentiator $(G(s))$ for different methods. 
Table 4. Numerical and stability analysis of $G(s)$.

\begin{tabular}{|c|c|c|c|c|}
\hline Technique & $t_{1} \in(0,125)$ & $t_{2} \in(0,250)$ & $\mathrm{H}_{\infty}$-norm & Stability \\
\hline \hline Oustaloup & 0.0315 & 0.0213 & 6.0256 & stable \\
refined Oustaloup & 0.0493 & 0.0439 & 7.9227 & stable \\
Matsuda & 0.0363 & 0.0266 & 8.2832 & stable \\
proposed & 0.0368 & 0.0212 & 7.1467 & stable \\
\hline
\end{tabular}

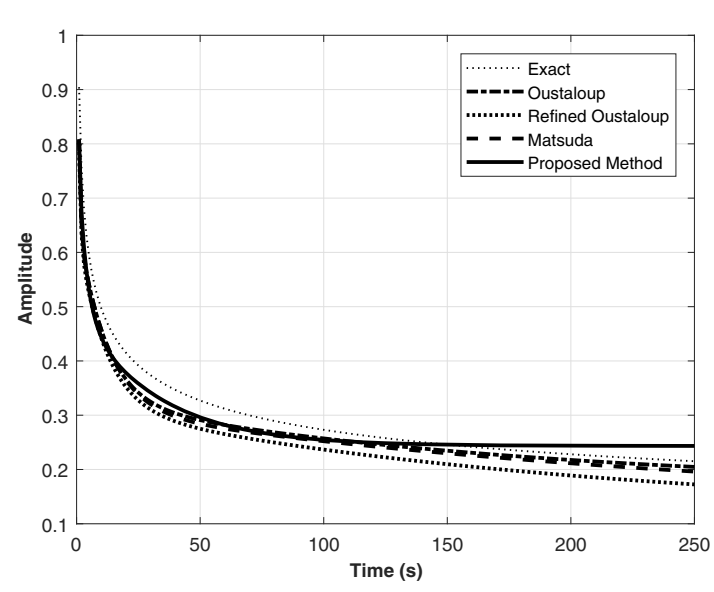

Fig. 8. Step responses of the fractional-order differentiator $(G(s))$ for different methods.

proposed approach has the least error of 0.0212 . This is an indication that, for longer time periods, the proposed approach yields a better approximation than the other approaches. Furthermore, the stability analysis of $G(s)$ for different methods given in Table 4 shows that all the approximation techniques are stable with a finite value of the $\mathrm{H}_{\infty}$-norm.

4.2. Fractional-order integrator. In this example, the proposed approach is demonstrated for a fractional-order integrator given by

$$
P(s)=\frac{1}{s^{0.6}} .
$$

Here, a similar comparison is made as to the differentiator example of Section 4.1. Hence, the approximated transfer function model using the proposed approach is

$$
\begin{gathered}
s^{5}+2134 s^{4}+2.094 \times 10^{5} s^{3} \\
P(s) \approx \frac{+1.582 \times 10^{6} s^{2}+1.3 \times 10^{6} s+8.884 \times 10^{4}}{148.8 s^{5}+4.646 \times 10^{4} s^{4}+9.294 \times 10^{5} s^{3}} . \\
+1.828 \times 10^{6} s^{2}+3.731 \times 10^{5} s+2353
\end{gathered}
$$

while those using other methods are given in Appendix B The frequency response plots of the proposed approach
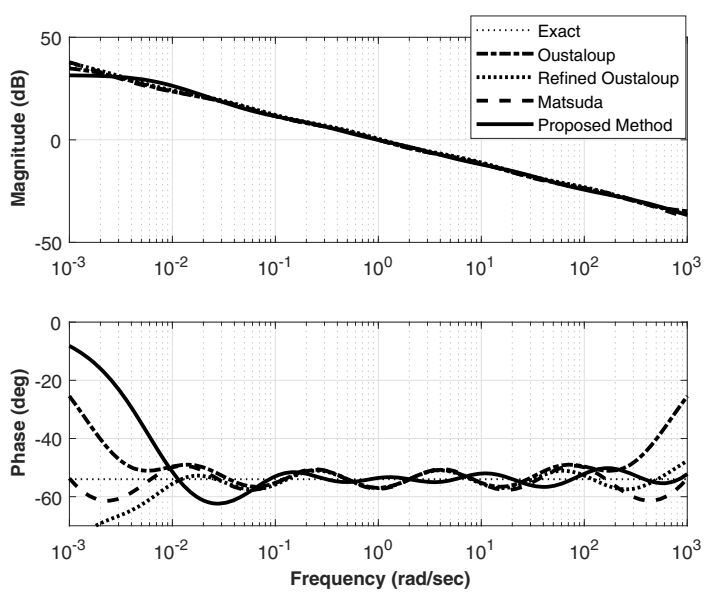

Fig. 9. Bode plots of the fractional-order integrator $(P(s))$ for different methods.

in comparison with the Oustaloup, refined Oustaloup and Matsuda approaches are presented in Fig. 9. From the figure, it can be observed that, compared with the other three techniques, the proposed method is more accurate. Furthermore, in the time domain, the step response of all the compared approaches is shown in Fig. 10 while the numerical assessment is given in Table 5. It can be observed from both the response and the table that, for the longest time range $t \in(0,250)$, the proposed approach has the least error of 0.1146 while, for the shorter time range $t \in(0,125)$, the Matsuda technique has the least error of 0.1280 . This indicates that the proposed approach produces a better approximation for longer time periods than the other approaches. This is in agreement with the case of the fractional-order differentiator given in Section 4.1 Furthermore, the stability analysis of various methods also given in Table 5 shows that the approximation transfer functions of the Oustaloup, Matsuda and proposed approach are stable, with the proposed technique having the least $\mathrm{H}_{\infty}$-norm of 37.7568. From the table, it can also be seen that the approximated transfer function using the refined Oustaloup is unstable.

4.3. Fractional-order $\mathrm{PID}$ controller $\left(\mathrm{PI}^{\lambda} \mathrm{D}^{\mu}\right)$. In this example, the proposed approach is demonstrated for the $\mathrm{PI}^{\lambda} \mathrm{D}^{\mu}$ controller, which consists of both the 
Table 5. Numerical and stability analysis of $P(s)$.

\begin{tabular}{|c|c|c|c|c|}
\hline Technique & $t_{1} \in(0,125)$ & $t_{2} \in(0,250)$ & $\mathrm{H}_{\infty}$-norm & Stability \\
\hline \hline Oustaloup & 0.2531 & 1.0345 & 63.0957 & stable \\
refined Oustaloup & 1.2426 & 2.1651 & inf & unstable \\
Matsuda & 0.1280 & 0.3347 & 156.4489 & stable \\
proposed & 0.8510 & 0.1146 & 37.7568 & stable \\
\hline
\end{tabular}

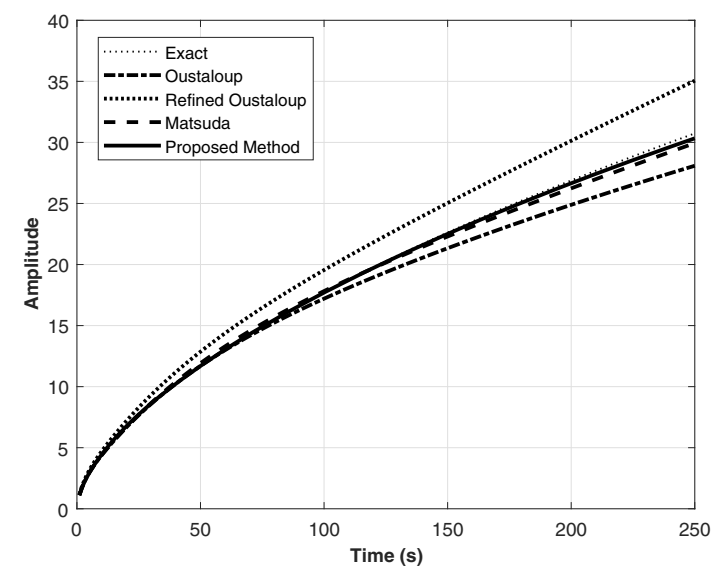

Fig. 10. Step responses of the fractional-order integrator $(P(s))$ for different methods.

fractional-order differentiator and the integrator given in Sections 4.1 and 4.2. respectively. The transfer function of the $\mathrm{PI}^{\lambda} \mathrm{D}^{\mu}$ controller used for demonstration is

$$
\begin{gathered}
C(s)=5+\frac{1}{s^{0.8}}+2 s^{0.5} \\
1.64 \times 10^{5} s^{10}+1.122 \times 10^{8} s^{9} \\
+2.207 \times 10^{10} s^{8}+9.906 \times 10^{11} s^{7} \\
+1.546 \times 10^{13} s^{6}+7.506 \times 10^{13} s^{5} \\
+1.384 \times 10^{14} s^{4}+9.075 \times 10^{13} s^{3} \\
+2.321 \times 10^{13} s^{2}+1.953 \times 10^{12} s \\
+5.153 \times 10^{10} \\
+s) \approx \\
1379 s^{10}+2.47 \times 10^{6} s^{9} \\
+8.25 \times 10^{8} s^{8}+6.329 \times 10^{10} s^{7} \\
+1.405 \times 10^{12} s^{6}+8.811 \times 10^{12} s^{5} \\
+1.842 \times 10^{13} s^{4}+1.202 \times 10^{13} s^{3} \\
+2.418 \times 10^{12} s^{2}+1.009 \times 10^{11} s \\
+3.871 \times 10^{8}
\end{gathered}
$$

For this example, approximation will be performed in two stages. First, the fractional-order differentiator term $\left(s^{0.5}\right)$ and the fractional-order integrator term $\left(1 / s^{0.8}\right)$ will be approximated using the approximation table given in Table 2 Then, using the approximated
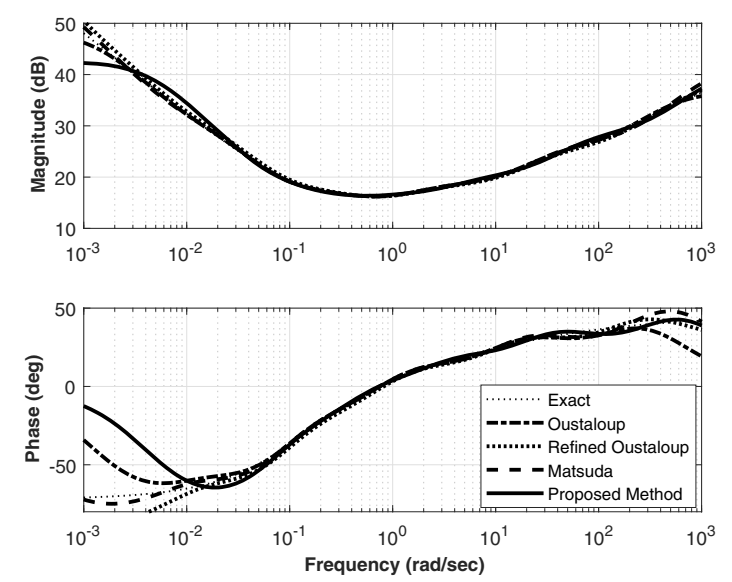

Fig. 11. Bode plots of the fractional-order PID controller $(C(s))$ for different methods.

transfer functions, the overall approximation of $C(s)$ is done by combining this integral with differential terms, which will lead to the transfer function of $C(s)$ given in (37).

Therefore, the frequency plot of the approximated $C(s)$ using the proposed approach in comparison with the Oustaloup, refined Oustaloup and Matsuda techniques is presented in Fig. 11, while the stability analysis of the figure is given in Table 6. From both the figure and the table, it can be observed that, compared with the other three techniques, the proposed method is more accurate. Furthermore, the stability analysis shows that the approximation transfer functions of the Oustaloup, Matsuda and proposed approach are stable and that of the refined Oustaloup technique is unstable. From the table, it can also be seen that the proposed technique has the least $\mathrm{H}_{\infty}$-norm of 133.1226 .

The result obtained here shows an improvement regarding the separate integrator and differentiator cases. This indicates that combining the two using the proposed approach yields an overall better result. This will, in turn, lead to an overall improvement in system performance.

4.4. Fractional-order low-pass filter. Apart from fractional-order controllers, the proposed approach can also be used to approximate the other fractional-order 
Table 6. Stability analysis of $C(s)$ for various methods.

\begin{tabular}{|c|c|c|}
\hline Technique & $\mathrm{H}_{\infty}$-norm & Stability \\
\hline \hline Oustaloup & 256.2519 & stable \\
refined Oustaloup & $\inf$ & unstable \\
Matsuda & $1.2269 \times 10^{3}$ & stable \\
proposed & 133.1226 & stable \\
\hline
\end{tabular}

Table 7. Stability analysis of $F(s)$ for various methods.

\begin{tabular}{|c|c|c|c|}
\hline \multirow{2}{*}{ Technique } & \multicolumn{2}{|c|}{ H-norms } & \multirow{2}{*}{ Stability } \\
\cline { 2 - 3 } & $\mathrm{H}_{2}$-norm & $\mathrm{H}_{\infty}$-norm & \\
\hline \hline Oustaloup & 0.9773 & 1.0273 & stable \\
refined Oustaloup & 0.9841 & 1.0276 & stable \\
Matsuda & 0.9772 & 1.0275 & stable \\
proposed & 0.9784 & 1.0267 & stable \\
\hline
\end{tabular}

systems such as the fractional-order low-pass filter

$$
F(s)=\frac{1}{0.5 s^{1.15}+1} .
$$

The details of the filter transfer function are available in the work of Kishore et al. (2017). Consequently, the approximated transfer function using the proposed approach is

$$
\begin{array}{r}
s^{5}+387.7 s^{4}+6767 s^{3}+1.119 \times 10^{4} s^{2} \\
+2004 s+19.27 \\
+1.181 \times 10^{4} s^{2}+2008 s+19.27
\end{array}
$$

Here, the approximation of $F(s)$ will be performed in three stages. First, based on (30), the fractional-order derivative $s^{1.15}$ is divided into $s \times s^{0.15}$. Then, $s^{0.15}$ is approximated using the proposed approach. Finally, by substituting this in (38), the overall approximated transfer function of $F(s)$ is determined.

The frequency plots of the approximated filter using the proposed approach in comparison with the Oustaloup, refined Oustaloup and Matsuda approaches are presented in Fig. 12, while the stability analysis related to the figure is given in Table 7. From the results, it can be seen that, just as in the case of a $\mathrm{PI}^{\lambda} \mathrm{D}^{\mu}$ controller, the proposed method is more accurate compared with the other three techniques. Furthermore, the stability analysis also shows that all the compared techniques performed better and stable with $\mathrm{H}_{2}$-and $\mathrm{H}_{\infty}$-norms of around 0.98 and 1.02 respectively.

4.5. Fractional-order transfer function (FOTF). To further demonstrate the effectiveness of the proposed approach, a fractional-order transfer function (FOTF) reported by Khanra et al. $(2011 ; 2013)$ is considered. The
FOTF in polynomial form with fractional powers is

$$
R(s)=\frac{s+1}{10 s^{3.2}+185 s^{2.5}+288 s^{0.7}+1} .
$$

Like in the previous case of $F(s)$, approximation will be performed in three stages. First, based on (30), the fractional-order derivatives $s^{3.2}$ and $s^{2.5}$ will be factorized as $s^{3} \times s^{0.2}$ and $s^{2} \times s^{0.5}$, respectively. Then $s^{0.2}, s^{0.5}$ and $s^{0.7}$ will be approximated using Table 2, Finally, by substituting all these in (40), the overall approximated transfer function of $R(s)$ is determined. Thus, the approximated transfer function of $R(s)$ is

$$
\begin{aligned}
& s^{16}+5206 s^{15}+7.573 \times 10^{6} s^{14} \\
& +3.565 \times 10^{9} s^{13}+5.807 \times 10^{11} s^{12} \\
& +3.69 \times 10^{13} s^{11}+9.463 \times 10^{14} s^{10} \\
& +1.085 \times 10^{16} s^{9}+5.936 \times 10^{16} s^{8} \\
& +1.626 \times 10^{17} s^{7}+2.361 \times 10^{17} s^{6} \\
& +1.854 \times 10^{17} s^{5}+7.647 \times 10^{16} s^{4} \\
& +1.53 \times 10^{16} s^{3}+1.359 \times 10^{15} s^{2} \\
& +4.618 \times 10^{13} s+4.001 \times 10^{11} \\
& 44.31 s^{18}+2.31 \times 10^{5} s^{17}+3.299 \times 10^{8} s^{16} \\
& +1.355 \times 10^{11} s^{15}+2.312 \times 10^{13} s^{14} \\
& +1.738 \times 10^{15} s^{13}+5.26 \times 10^{16} s^{12} \\
& +7.555 \times 10^{17} s^{11}+5.299 \times 10^{18} s^{10} \\
& +1.788 \times 10^{19} s^{9}+3.458 \times 10^{19} s^{8} \\
& +4.593 \times 10^{19} s^{7}+4.372 \times 10^{19} s^{6} \\
& +2.484 \times 10^{19} s^{5}+6.997 \times 10^{18} s^{4} \\
& +8.796 \times 10^{17} s^{3}+3.93 \times 10^{16} s^{2} \\
& +5.396 \times 10^{14} s+2.125 \times 10^{12}
\end{aligned}
$$

The frequency plots of $R(s)$ using the proposed approach in comparison with the Oustaloup, refined the Oustaloup and Matsuda techniques are presented in Fig. 13, while the stability analysis related to the figure is given in Table 8. From the results, it can be seen that all the compared techniques have produced the best approximation. Furthermore, the stability analysis from the table also shows that all the approximated transfer functions are stable with the proposed approach having the least $\mathrm{H}_{2}$ and $\mathrm{H}_{\infty}$-norms of 0.0138 and 0.1883 , respectively.

To further evaluate the performance of the proposed approach, comparison with the power series expansion technique is made. An example is a finite impulse response (FIR) filter for a discretized fractional-order differentiator based on PSE, given in Appendix C. Thus, the Bode plot of the proposed approach in 

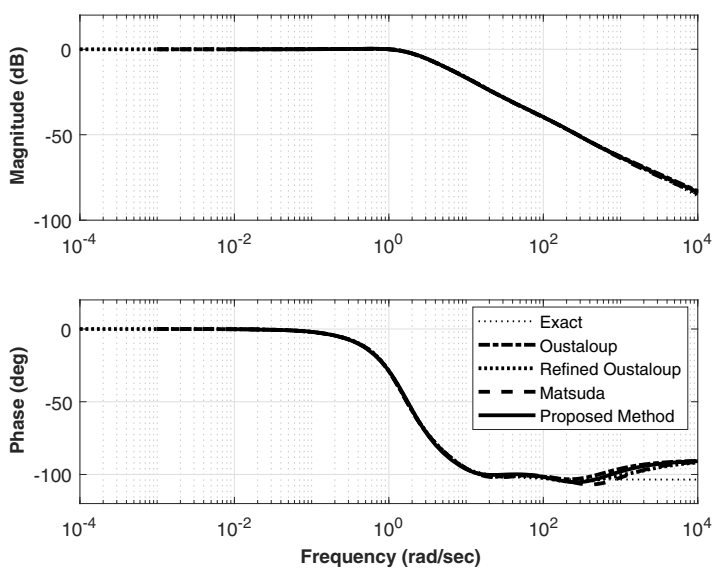

Fig. 12. Bode plots of the fractional-order low-pass filter $(F(s))$ for different methods.
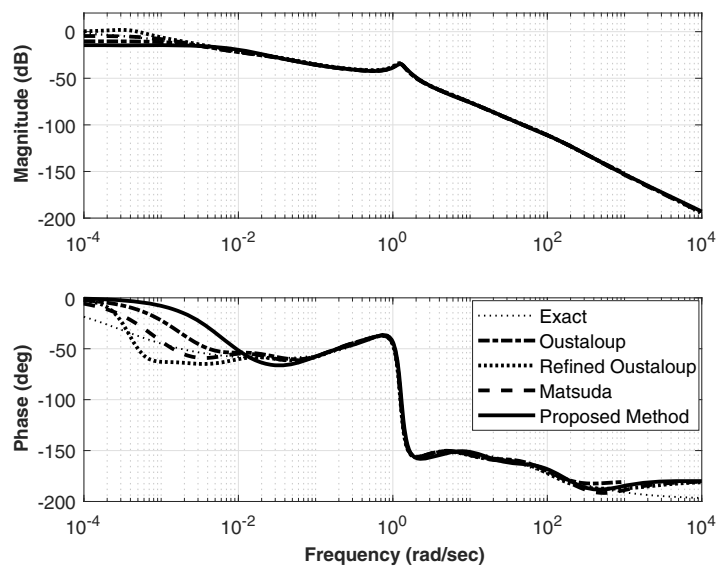

Fig. 13. Bode plots of FOTF $(R(s))$ for different methods.

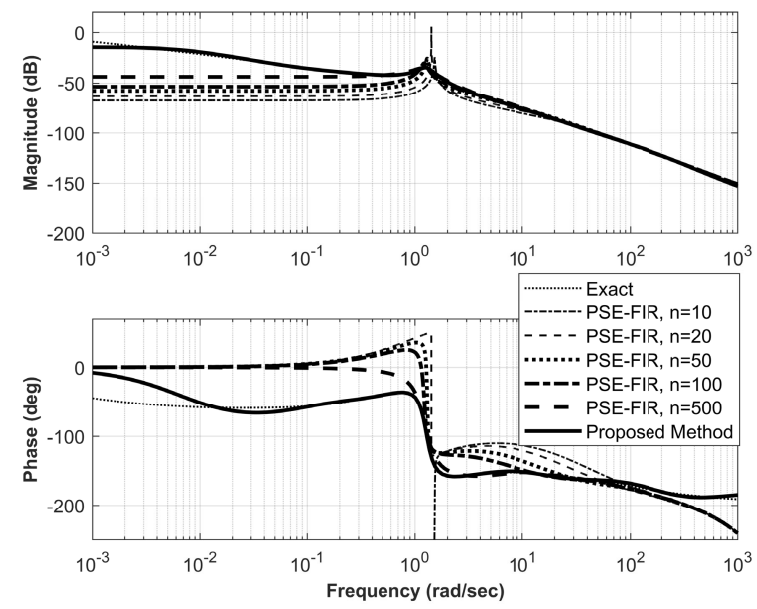

Fig. 14. Bode plots of the proposed approach compared with PSE-FIR for $T=0.001$ and various values of $n$.
Table 8. Stability analysis of $R(s)$ for various methods.

\begin{tabular}{|c|c|c|c|}
\hline \multirow{2}{*}{ Technique } & \multicolumn{2}{|c|}{ H-norms } & \multirow{2}{*}{ Stability } \\
\cline { 2 - 3 } & $\mathrm{H}_{2}$-norm & $\mathrm{H}_{\infty}$-norm & \\
\hline \hline Oustaloup & 0.0141 & 0.3042 & stable \\
Refined Oustaloup & 0.0225 & 1.2395 & stable \\
Matsuda & 0.0164 & 0.5885 & stable \\
Proposed & 0.0138 & 0.1883 & stable \\
\hline
\end{tabular}

Table 9. Stability analysis of $R(s)$ with PSE-FIR for various values of $n$

\begin{tabular}{|c|c|c|c|c|}
\hline \multirow{2}{*}{ Technique } & \multirow{2}{*}{ Order } & \multicolumn{2}{|c|}{ H-norms } & \multirow{2}{*}{ Stability } \\
\cline { 3 - 4 } & & $\mathrm{H}_{2}$-norm & $\mathrm{H}_{\infty}$-norm & \\
\hline \hline & 10 & inf & inf & unstable \\
PSE-FIR & 20 & 0.0024 & 7.0452 & stable \\
& 50 & 0.0002 & 0.0508 & stable \\
& 100 & 0.0002 & 0.0301 & stable \\
& 500 & 0.0002 & 0.0176 & stable \\
Proposed & 5 & 0.0138 & 0.1883 & stable \\
\hline
\end{tabular}

comparison with the FIR filter for orders of truncation or approximation of 10, 20, 50, 100 and 500 is shown in Fig. 14. The stability analysis of the figure is given in Table 9 From the figure and the table, it can be seen that the proposed approach performs better compared with the PSE based FIR filter. Observing the responses, it can also be seen that, for orders less than 10, the transfer function using PSE-FIR is unstable and, for an order greater then 50, PSE-FIR approaches the exact response, thereby generating a very high integer-order transfer function.

\section{Conclusion}

In this paper, a simple curve fitting approximation technique for the fractional-order differintegral operator using the frequency response was proposed. With the approach, an approximation table for the fractional-order differentiator was obtained. The table can be used directly to generate approximated transfer functions of fractional-order based controllers and systems. Results from the simulation study show that the proposed approach produced better approximation of the fractional-order parameters within the desired frequency range when compared with the Oustaloup, refined Oustaloup and Matsuda approximations. Furthermore, time domain analysis of the results shows that the proposed approach gives better approximation for longer time periods than the Oustaloup, refined Oustaloup and Matsuda approximations. The stability analysis in terms of the $\mathrm{H}_{2}$ and $\mathrm{H}_{\infty}$-norms also confirms that the proposed approach is better and stable.

As part of future studies, an attempt will be made to implement a fractional-order controller designed using the proposed approach on a real-time plant. 


\section{Acknowledgment}

This work was supported by Universiti Teknologi PETRONAS through the Yayasan UTP Fundamental Research Grant 0153AA-H16.

\section{References}

Atherton, D.P., Tan, N. and Yüce, A. (2014). Methods for computing the time response of fractional-order systems, IET Control Theory \& Applications 9(6): 817-830.

Balas, G., Chiang, R., Packard, A. and Safonov, M. (2007). Robust Control Toolbox 3: User' Guide, MathWorks, Natick, MA.

Bingi, K., Ibrahim, R., Karsiti, M.N., Hassan, S.M. and Harindran, V.R. (2018a). A comparative study of 2DOF PID and 2DOF fractional order PID controllers on a class of unstable systems, Archives of Control Sciences 28(4): 635-682.

Bingi, K., Ibrahim, R., Karsiti, M.N., Hassan, S.M. and Harindran, V.R. (2018b). Real-time control of pressure plant using 2DOF fractional-order PID controller, Arabian Journal for Science and Engineering 44(3): 2091-2102.

Caponetto, R. (2010). Fractional Order Systems: Modeling and Control Applications, World Scientific, Singapore.

Das, S. (2011). Functional Fractional Calculus, Springer, Berlin/Heidelberg.

de Oliveira Valério, D.P.M. (2005). Fractional Robust System Control, PhD thesis, Universidade Técnica de Lisboa, Lisboa.

Deniz, F.N., Alagoz, B.B., Tan, N. and Atherton, D.P. (2016). An integer order approximation method based on stability boundary locus for fractional order derivative/integrator operators, ISA Transactions 62: 154-163.

Djouambi, A., Charef, A. and Besançon, A.V. (2007). Optimal approximation, simulation and analog realization of the fundamental fractional order transfer function, International Journal of Applied Mathematics and Computer Science 17(4): 455-462, DOI: 10.2478/v10006-007-0037-9.

Du, B., Wei, Y., Liang, S. and Wang, Y. (2017). Rational approximation of fractional order systems by vector fitting method, International Journal of Control, Automation and Systems 15(1): 186-195.

Joice Nirmala, R. and Balachandran, K. (2017). The controllability of nonlinear implicit fractional delay dynamical systems, International Journal of Applied Mathematics and Computer Science 27(3): 501-513, DOI: 10.1515/amcs-2017-0035.

Kaczorek, T. (2018). Decentralized stabilization of fractional positive descriptor continuous-time linear systems, International Journal of Applied Mathematics and Computer Science 28(1): 135-140, DOI: 10.2478/amcs-2018-0010.

Khanra, M., Pal, J. and Biswas, K. (2011). Rational approximation and analog realization of fractional order differentiator, 2011 International Conference on Process Automation, Control and Computing (PACC), Coimbatore, India, pp. 1-6.
Khanra, M., Pal, J. and Biswas, K. (2013). Rational approximation and analog realization of fractional order transfer function with multiple fractional powered terms, Asian Journal of Control 15(3): 723-735.

Kishore, B., Ibrahim, R., Karsiti, M.N. and Hassan, S.M. (2017). Fractional-order filter design for set-point weighted PID controlled unstable systems, International Journal of Mechanical \& Mechatronics Engineering 17(5): 173-179.

Kishore, B., Ibrahim, R., Karsiti, M.N. and Hassan, S.M. (2018). Fractional order set-point weighted PID controller for $\mathrm{pH}$ neutralization process using accelerated PSO algorithm, Arabian Journal for Science and Engineering 43(6): 2687-2701.

Krajewski, W. and Viaro, U. (2011). On the rational approximation of fractional order systems, 16th International Conference on Methods and Models in Automation and Robotics (MMAR), Międzyzdroje, Poland, pp. 132-136.

Krajewski, W. and Viaro, U. (2014). A method for the integer-order approximation of fractional-order systems, Journal of the Franklin Institute 351(1): 555-564.

Krishna, B. (2011). Studies on fractional order differentiators and integrators: A survey, Signal Processing 91(3): 386-426.

Li, Z., Liu, L., Dehghan, S., Chen, Y. and Xue, D. (2017). A review and evaluation of numerical tools for fractional calculus and fractional order controls, International Journal of Control 90(6): 1165-1181.

Liang, S., Peng, C., Liao, Z. and Wang, Y. (2014). State space approximation for general fractional order dynamic systems, International Journal of Systems Science 45(10): 2203-2212.

Meng, L. and Xue, D. (2012). A new approximation algorithm of fractional order system models based optimization, Journal of Dynamic Systems, Measurement, and Control 134(4): 044504.

Merrikh-Bayat, F. (2012). Rules for selecting the parameters of Oustaloup recursive approximation for the simulation of linear feedback systems containing PI $\lambda \mathrm{D} \mu$ controller, Communications in Nonlinear Science and Numerical Simulation 17(4): 1852-1861.

Mitkowski, W. and Oprzedkiewicz, K. (2016). An estimation of accuracy of Charef approximation, in S. Domek and P. Dworak (Eds.), Theoretical Developments and Applications of Non-Integer Order Systems, Springer, Berlin/Heidelberg, pp. 71-80.

Monje, C.A., Chen, Y., Vinagre, B.M., Xue, D. and Feliu-Batlle, V. (2010). Fractional-Order Systems and Controls: Fundamentals and Applications, Springer, Berlin/Heidelberg.

Oprzedkiewicz, K. (2014). Approximation method for a fractional order transfer function with zero and pole, Archives of Control Sciences 24(4): 447-463.

Oustaloup, A., Levron, F., Mathieu, B. and Nanot, F.M. (2000). Frequency-band complex noninteger differentiator: characterization and synthesis, IEEE Transactions on Circuits and Systems I: Fundamental Theory and Applications 47(1): 25-39. 
Pachauri, N., Singh, V. and Rani, A. (2018). Two degrees-of-freedom fractional-order proportional-integral-derivative-based temperature control of fermentation process, Journal of Dynamic Systems, Measurement, and Control 140(7): 071006.

Petráš, I. (2011a). Fractional derivatives, fractional integrals, and fractional differential equations in Matlab, in A. Assi (Ed.), Engineering Education and Research Using MAT$L A B$, InTech, London, pp. 239-264.

Petráš, I. (2011b). Fractional-Order Nonlinear Systems: Modeling, Analysis and Simulation, Springer, Berlin/Heidelberg.

Poinot, T. and Trigeassou, J.-C. (2003). A method for modelling and simulation of fractional systems, Signal processing 83(11): 2319-2333.

Shah, P. and Agashe, S. (2016). Review of fractional PID controller, Mechatronics 38: 29-41.

Sheng, H., Chen, Y. and Qiu, T. (2011). Fractional Processes and Fractional-Order Signal Processing: Techniques and Applications, Springer, Berlin/Heidelberg.

Shi, G. (2016). On the nonconvergence of the vector fitting algorithm, IEEE Transactions on Circuits and Systems II: Express Briefs 63(8): 718-722.

Tepljakov, A., Petlenkov, E. and Belikov, J. (2012). Application of Newton's method to analog and digital realization of fractional-order controllers, International Journal of Microelectronics and Computer Science 2(2): 45-52.

Valério, D., Trujillo, J.J., Rivero, M., Machado, J.T. and Baleanu, D. (2013). Fractional calculus: A survey of useful formulas, The European Physical Journal Special Topics 222(8): 1827-1846.

Vinagre, B., Podlubny, I., Hernandez, A. and Feliu, V. (2000). Some approximations of fractional order operators used in control theory and applications, Fractional Calculus and Applied Analysis 3(3): 231-248.

Wei, Y., Gao, Q., Peng, C. and Wang, Y. (2014a). A rational approximate method to fractional order systems, International Journal of Control, Automation and Systems 12(6): 1180-1186.

Wei, Y., Gao, Q., Peng, C. and Wang, Y. (2014b). A rational approximate method to fractional order systems, International Journal of Control, Automation and Systems 12(6): 1180-1186.

Xue, D. (2017). Fractional-order Control Systems: Fundamentals and Numerical Implementations, Walter de Gruyter $\mathrm{GmbH}$, Berlin.

Xue, D., Chen, Y. and Attherton, D.P. (2007). Linear Feedback Control: Analysis and Design with MATLAB, SIAM, Philadelphia, PA.

Xue, D., Zhao, C. and Chen, Y. (2006). A modified approximation method of fractional order system, Proceedings of the 2006 IEEE International Conference on Mechatronics and Automation, Luoyang, China pp. 1043-1048.
Yüce, A., Deniz, F.N. and Tan, N. (2017). A new integer order approximation table for fractional order derivative operators, IFAC-PapersOnLine 50(1): 9736-9741.

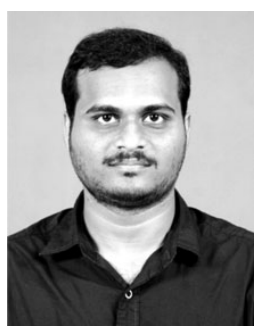

Kishore Bingi received the BTech degree (Hons) in electrical and electronics engineering from Bapatla Engineering College, Bapatla, India, in 2012, and the MTech degree (Hons) in instrumentation and control systems from the National Institute of Technology Calicut, Kerala, India, in 2014. He is currently pursuing the $\mathrm{PhD}$ degree with the Electrical and Electronic Engineering Department, Universiti Teknologi PETRONAS, Perak, Malaysia. He was with Tata Consultancy Service as an assistant systems engineer from 2015 to 2016. His current research interests include process modeling, control, and optimization.

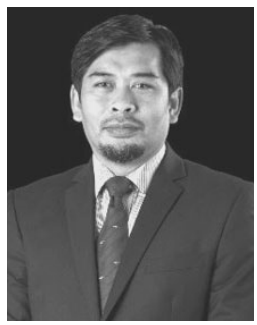

Rosdiazli Ibrahim received the BEng degree in electrical engineering from Universiti Putra Malaysia, Kembangan, Malaysia, in 1996, the MSc degree in automation and control from Newcastle University, UK, in 2000, and the PhD degree in electrical and electronic engineering from the University of Glasgow, UK, in 2008. He is an associate professor with the Department of Electrical and Electronics Engineering at Universiti Teknologi PETRONAS (UTP), Seri Iskandar, Perak, Malaysia. He is currently the dean at the Centre for Graduate Studies, UTP. His present research interests include intelligent control and non-linear multi-variable process modeling for control application.

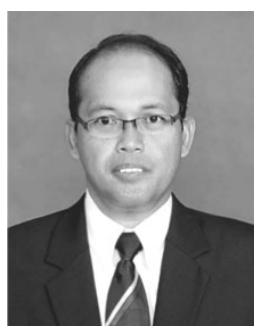

Mohd Noh Karsiti received the BEng degree in electrical engineering from California State University, Long Beach, CA, in 1985, the MSc degree in electrical engineering (control) from California State University in 1987, and the $\mathrm{PhD}$ degree in electrical and computer engineering (hierarchical control) from the University of California, Irvine, CA, in 1991. He joined Universiti Teknologi PETRONAS, Perak, Malaysia, in 1999 , where he is currently an associate professor. His present research interests include advanced process control, robotics systems manipulator design and control, control of highly nonlinear systems and automatic compensation of control systems.

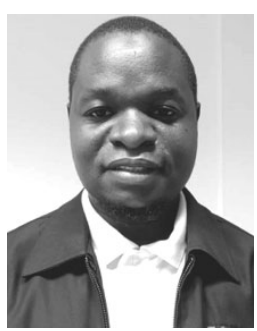

Hassan Sabo Miya received the BEng degree (Hons) in electrical and electronic engineering from Abubakar Tafawa Balewa University, Bauchi, Nigeria, in 2008, the MSc degree (Hons) in control systems from the University of Sheffield, UK, in 2011, and the PhD degree at the Electrical and Electronic Engineering Department, Universiti Teknologi PETRONAS, Perak, Malaysia. He is currently a lecturer in the Department of Electrical and Electronic Engineering, Abubakar Tafawa Balewa University, Bauchi, Nigeria. His present research interests include wireless networked control systems, intelligent control, soft computing, and optimization. 


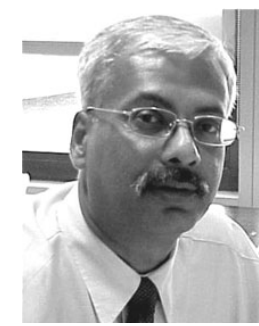

Vivekananda Rajah Harindran received the BSc degree in electrical and electronics engineering from Huddersfield University, UK, in 1984 and the MSc degree in technology management from Staffordshire University, UK, in 2010. He joined PETRONAS Group Technical Solutions in 1992, where he is currently the custodian engineer in the Division of Instrumentation and Control. He has 27 years' experience in petrochemical, oil and gas industries, with skills in project design, improving plant production, reliability and availability. His current research interests include the design and implementation of instrumentation systems and control.

\section{Appendix A}

\section{MATLAB code for the proposed curve fitting based approximation}

\% Curve Fitting Approach for $s^{\gamma} \%$

function Gp=curveFitting (gam, N,wl,wh)

$s=\operatorname{tf}\left({ }^{\prime} s '\right)$;

$F R D=f r d(s,(l o g s p a c e(\log 10(w 1), \ldots$

$$
\log 10(w h)))) \text {; }
$$

FRD. ResponseData=FRD. ResponseData .^gam;

$\mathrm{Gp}=\mathrm{fitfrd}(\mathrm{FRD}, \mathrm{N})$;

[num, den] =ss2tf (Gp.A, Gp.B, Gp.C, Gp.D) ;

$\mathrm{Gp}=\operatorname{tf}(\mathrm{num}, \mathrm{den})$; end

To compute approximation with the help of above-proposed curve fitting approximation, the MATLAB Robust Control Toolbox is required. The user guide of the toolbox is available in the work of Balas et al. (2007).

\section{Appendix B}

\section{Results from other approximation methods}

The approximated transfer function of the fractional-order differentiator given in Section 4.1 using the Oustaloup $G_{O}(s)$, refined Oustaloup $G_{R}(s)$ and Matsuda $G_{M}(s)$ approximation techniques is presented in (B1), (B2) and (B3), respectively. Similarly, for the fractional-order integrator given in Section 4.2 the approximated transfer functions using the Oustaloup $P_{O}(s)$, refined Oustaloup $P_{R}(s)$ and Matsuda $P_{M}(s)$ approximation techniques are

$$
\begin{aligned}
G_{O}(s) \approx & \frac{6.026 s^{5}+1128 s^{4}+1.253 \times 10^{4} s^{3}}{s^{5}+384 s^{4}+8750 s^{3}} \\
& +1.253 \times 10^{4} s^{2}+1128 s+6.026
\end{aligned}
$$

$$
\begin{aligned}
& 7.923 s^{13}+1.029 \times 10^{4} s^{12} \\
& +1.664 \times 10^{6} s^{11}+1.832 \times 10^{7} s^{10} \\
& +1.279 \times 10^{7} s^{9}+5.634 \times 10^{5} s^{8} \\
& +1566 s^{7}+0.2747 s^{6} \\
& +3.039 \times 10^{-6} s^{5}+2.122 \times 10^{-12} s^{4} \\
& +9.347 \times 10^{-20} s^{3}+2.588 \times 10^{-28} s^{2} \\
& G_{R}(s) \approx \frac{+4.252 \times 10^{-38} s}{s^{13}+1885 s^{12}+5.853 \times 10^{5} s^{11}}, \\
& +1.315 \times 10^{7} s^{10}+1.883 \times 10^{7} s^{9} \\
& +1.705 \times 10^{6} s^{8}+1.01 \times 10^{4} s^{7} \\
& +5.758 s^{6}+0.0008956 s^{5} \\
& +1.864 \times 10^{-8} s^{4}+2.655 \times 10^{-14} s^{3} \\
& +2.398 \times 10^{-21} s^{2}+1.362 \times 10^{-29} s \\
& +4.589 \times 10^{-39} \\
& 8.283 s^{5}+2347 s^{4}+2.872 \times 10^{4} s^{3} \\
& G_{M}(s) \approx \frac{+1.982 \times 10^{4} s^{2}+749.1 s+1}{s^{5}+749.1 s^{4}+1.982 \times 10^{4} s^{3}}, \\
& +2.872 \times 10^{4} s^{2}+2347 s+8.283 \\
& s^{5}+614.2 s^{4}+2.239 \times 10^{4} s^{3} \\
& P_{O}(s) \approx \frac{+5.129 e 04 s^{2}+7384 s+63.1}{63.1 s^{5}+7384 s^{4}+5.129 \times 10^{4} s^{3}}, \\
& +2.239 e 04 s^{2}+614.2 s+1 \\
& s^{13}+3392 s^{12}+1.728 \times 10^{6} s^{11} \\
& +6.224 \times 10^{7} s^{10}+1.426 \times 10^{8} s^{9} \\
& +2.068 \times 10^{7} s^{8}+1.99 \times 10^{5} s^{7} \\
& +209.6 s^{6}+0.0623 s^{5} \\
& +2.129 \times 10^{-6} s^{4}+4.857 \times 10^{-12} s^{3} \\
& +7.017 \times 10^{-19} s^{2}+6.374 \times 10^{-27} s \\
& P_{R}(s) \approx \frac{+3.436 \times 10^{-36}}{148.1 s^{13}+1.819 \times 10^{5} s^{12}}, \\
& +1.938 \times 10^{7} s^{11}+1.338 \times 10^{8} s^{10} \\
& +5.84 \times 10^{7} s^{9}+1.608 \times 10^{6} s^{8} \\
& +2795 s^{7}+0.3065 s^{6} \\
& +2.12 \times 10^{-6} s^{5}+9.255 \times 10^{-13} s^{4} \\
& +2.548 \times 10^{-20} s^{3}+4.411 \times 10^{-29} s^{2} \\
& +4.532 \times 10^{-39} s
\end{aligned}
$$




$$
\begin{aligned}
& s^{5}+1807 s^{4}+8.141 \times 10^{4} s^{3} \\
&+1.922 \times 10^{5} s^{2}+2.565 \times 10^{4} s \\
& P_{M}(s) \approx+156.4 \\
& 156.4 s^{5}+2.565 \times 10^{4} s^{4} \\
&+1.922 \times 10^{5} s^{3}+8.141 \times 10^{4} s^{2} \\
&+1807 s+1
\end{aligned}
$$

\section{Appendix C}

Approximation of fractional-order differentiator using finite impulse response (FIR) based on PSE

According to Petráš (2011b; 2011a) and Caponetto (2010), the approximation of the discretized fractional-order differentiator in the form of the FIR filter based on PSE is as follows:

$$
\begin{aligned}
{ }_{0} \mathcal{D}_{T}^{\gamma} X(z) & =\left(\frac{1}{T}\right)^{\gamma} \operatorname{PSE}\left\{\left(1-z^{-1}\right)^{\gamma}\right\}_{n} \\
& \approx T^{-\gamma} R_{n}\left(z^{-1}\right)
\end{aligned}
$$

where

- $n$ is the order of approximation or truncation,

- $T$ is the sampling period,

- $R$ is the polynomial in the variable $z^{-1}$ of order, $n$ and

- $\operatorname{PSE}\left\{\left(1-z^{-1}\right)^{\gamma}\right\}$ denotes the power series expansion of the function $\left(1-z^{-1}\right)^{\gamma}$.

From (C1), it can be noted that the approximated power series expansion of $\left(1-z^{-1}\right)^{\gamma}$ in the form of the FIR filter has only zeros.
Received: 17 June 2018

Revised: 31 October 2018

Re-revised: 5 December 2018

Accepted: 8 January 2019 